\title{
Embellecimiento facial evolutivo dirigido por fuerzas
}

\author{
Ricardo Solano Monje ${ }^{1}$, Nayeli Joaquinita Meléndez Acosta², \\ Homero Vladimir Ríos Figueroa ${ }^{3}$ \\ ${ }^{1}$ Instituto Tecnológico Superior de Venustiano Carranza, Lázaro Cárdenas, \\ Pue. México \\ ${ }^{2}$ Universidad del Istmo, Campus Ixtepec, \\ Ixtepec, Oax., México \\ ${ }^{3}$ Universidad Veracruzana, Xalapa, \\ Ver., México \\ rsolano@itsvc.edu.mx, nayelimelendez@gmail.com, hrios@uv.com.mx
}

\begin{abstract}
Resumen. Este trabajo explica el desarrollo de una propuesta original para embellecer rostros, resultado de un algoritmo evolutivo y un algoritmo de dibujo estético de grafos. El algoritmo de dibujo estético de grafos opera en este contexto como el operador de mutación. La estrategia dirigida por fuerzas que es el fundamento del algoritmo de dibujo estético de grafos, hace uso de las leyes de Hooke que gobiernan la tensión en resortes. La solución de los resortes utiliza un modelo mecánico para producir diseños 2D "estéticamente agradables", calculando fuerzas repulsivas y atractivas entre nodos. En nuestro caso, los nodos que describen un grafo son una representación del rostro a embellecer. El objetivo de este trabajo es crear un rostro embellecido en comparación con el rostro de entrada. Un individuo $I$ ésta compuesto por la máscara del rostro de entrada, es decir Puntos de Referencia y Vector de Distancias. En los resultados experimentales se utilizó la base de datos de imágenes Fg-Net [7]. Nuestros resultados experimentales muestran rostros embellecidos usando nuestra aproximación, mostrando así una solución competitiva.
\end{abstract}

Palabras clave: Trazado estético de grafos, embellecimiento de rostros, representación de rostros por apariencia, eigenspaces de rostros.

\section{Force-directed Evolutionary Face Beautification}

\begin{abstract}
This work explains the development of a novel approach to beautify a face, merging an evolutionary approach and aesthetics graph drawing. The objective of this work is to build a beautify face seen against the input face. The aesthetics graph drawing algorithm works as the mutation operator. The approach of force-directed graph drawing which in turn is the solution to aesthetical graph drawing makes use of the law of Hooke about springs. The springs embedded approach uses a mechanical model to build 2D layouts aesthetically pleasing by computing attraction and repulsion forces in between nodes. In our case of face beautification, the graph nodes count as a representation of the face to be beautified. We have carried out tests using the Fg-Net face database [7]. Our
\end{abstract}


experimental results show beautified faces using our approach, meaning our approach as a competitive solution.

Keywords: Aesthetically pleasing graph layouts, face beautification, appearance representation of the face, modular eigenspaces of the face.

\section{Introducción}

Es perfectamente sano asumir que cualquier modelo que encontramos hoy en día en la portada de una revista ha sido manipulada por un especialista con habilidades de retoque. El desarrollo de una herramienta inteligente de embellecimiento tendrá impactos en cine y propaganda, entre muchos otros.

El embellecimiento facial por computadora es un tópico de Visión por Computadora. Este trabajo de investigación se centra únicamente en el aspecto de embellecer el rostro.

El contenido del artículo está organizado en 6 secciones de la siguiente manera: en la segunda sección se muestran algunos trabajos relacionados al problema del embellecimiento artificial. La tercera sección muestra el marco teórico del algoritmo de dibujo estético de grafos dirigido por fuerzas, utilizado como operador de mutación en el Algoritmo Evolutivo. En la cuarta sección se explica el funcionamiento del Algoritmo Evolutivo. La quinta sección muestra las pruebas y resultados obtenidos. Finalmente la sexta sección corresponde a las conclusiones.

\section{Embellecimiento del rostro}

El reconocimiento de rostro se lleva a cabo con información de bulto, por ello los detalles pueden ser perdonados o compensados cuando se ve el rostro por segunda vez y éste no es tan bello como la primera vez - la vez que se vio embellecido-. Es decir, que por analogía al reconocimiento de rostros, el embellecimiento artificial pasaría desapercibido hasta cierto punto.

El embellecimiento digital del rostros toma de entrada una fotografía -imagen frontal de un rostro y de forma automática mejora el atractivo del rostro e incrementa la calificación de belleza (la calificación predicha por la máquina de belleza, que califica el embellecimiento) del rostro.

Uno de los principales objetivos es lograr un rostro embellecido que mantenga una semejanza marcada - no dudosa - con el rostro original en una imagen de entrada por medio de algoritmos evolutivos.

En [3] Leyvand realiza la modificación y embellecimiento del rostro creando una máquina embellecedora construyendo una máscara con 84 puntos de referencia, con esta mascara se forma un vector de distancias entre los puntos de referencia que definen las 8 características faciales: las dos cejas, los dos ojos, el labio inferior y superior de la boca, la nariz y límites del rostro.

Eisenthal en [4] persigue en su trabajo calificar la belleza, es decir construye una máquina calificadora y solo localiza 37 puntos de referencia utilizando una SVM 
(Support Vector Machine), la cual es utiliza por Leyvand para calificar la belleza del rostro.

En [5] Solano realiza el embellecimiento del rostro y en [6] realizó una máquina calificadora, construye una máscara con 66 puntos de referencia. Esta mascara ha sido tomada como referencia en este trabajo. La Fig. 1(a) muestra los 66 puntos de referencia. La Fig. 1(b) las características faciales y la Fig. 1(c) la máscara formada por 89 distancias.

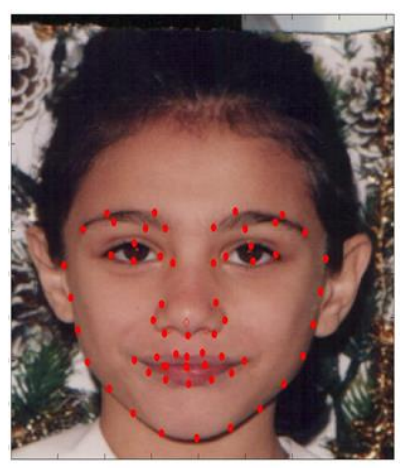

(a)

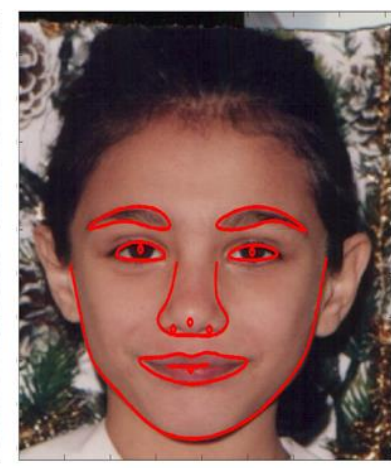

(b)

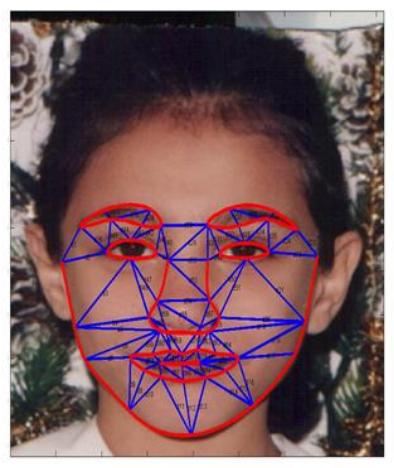

(c)

Fig. 1. (a) Los 66 puntos de referencia. (b) Las características faciales.

(c) Las 89 distancias entre puntos de referencia

\section{Algoritmo de dibujo estético de grafos dirigido por fuerzas}

Un grafo $\mathrm{G}=(\mathrm{V}, \mathrm{E})$ es un par dónde $V$ es el conjunto de vértices y $E$ es el conjunto de aristas. Un dibujo de un grafo $G$ en el plano es un mapeo $D$ de $V$ a $R$, dónde $R$ es el conjunto de números reales. Es decir, cada vértice $v$ se coloca en el punto $D(v)$ en el plano, y cada artista $(u, v)$ se muestra como un segmento de línea recta que conecta $D(u)$ y $D(v)$.

Los métodos de Fuerza dirigida construyen dibujos en línea recta de grafos generales utilizando un modelo físico en el que los vértices y las aristas del grafo son vistos como objetos físicos sujetos a varias fuerzas.

En 1984 el algoritmo de Eades [6] fue hecho para grafos con un máximo de 30 vértices y utiliza un modelo mecánico para producir diseños 2D "estéticamente agradables". El algoritmo se resume brevemente como sigue:

En esta versión, la fuerza resultante en cada nodo son la suma de las fuerzas atractivas (utilizando los nodos vecinos con arista adyacente) y de las repulsivas (calculada utilizando los nodos sin arista). Las fuerzas atractivas (entre nodos que tiene aristas) tienen la forma:

$$
f_{a}(u, v)=c_{1} \times \log \left(\frac{l}{c_{2}}\right)
$$

donde $c_{1}$ y $c_{2}$ son constantes y $l$ es la longitud de la arista. 
En tanto las fuerzas repulsivas (entre los nodos que no tienen aristas) quedan determinadas por la siguiente fórmula:

$$
f_{r}(u, v)=\left(\frac{c_{3}}{l^{2}}\right)
$$

donde $c_{3}$ es una constante y $l$ es la distancia entre nodos.

La fuerza $f(u)$ que experimenta un vértice $u$ es

$$
f(u)=\sum_{(u, v) \in E} f_{a}(u, v)+\sum_{(u, v) \notin E} f_{r}(u, v) .
$$

Algoritmo 1. Dibujo estético de grafos dirigido por fuerzas propuesto por Peter Eades (1984) A Heuristic for Graph Drawin.

Algoritmo (G: grafo);

Colocar los vértices de $\mathrm{G}$ en localizaciones aleatorias

Repetir M veces

Calcular la fuerza en cada vértice;

Mover el vértice $c_{4} *$ (Fuerza en el vértice);

Dibujar grafo;

Los valores $c_{1}=2, c_{2}=1, c_{3}=1$ y $c_{4}=0.1$, son apropiados para la mayoría de los grafos. La mayoría de los grafos alcanzan un estado de energía mínima después de 100 veces, es decir, $M=100$ [6].

Las Fig. 2 y 3 muestran la representación del algoritmo de dibujo estético de grafos dirigido por fuerzas enfocado al problema de esta investigación, embellecimiento digital del rostro por partes. En la Fig. 2 se observa la Boca modelada a través de resortes y en la Fig. 3 se observan las cejas modelas con resortes.

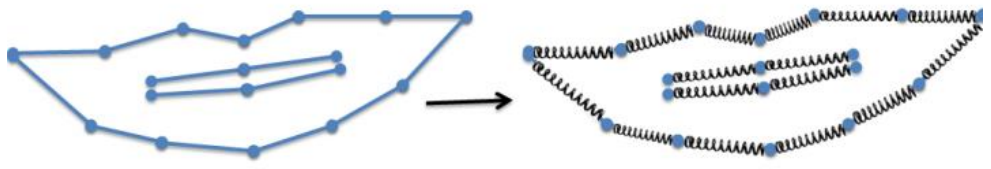

Fig. 2. La característica facial "La Boca", representación con resortes
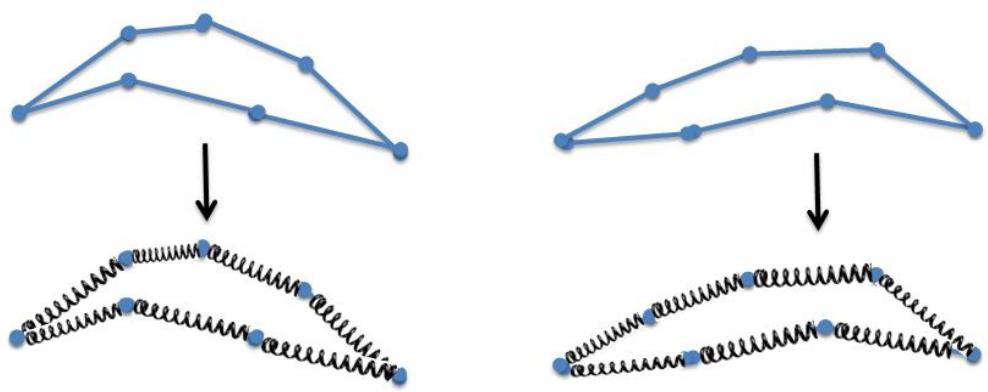

Fig. 3. La característica facial "Las Cejas", representación con resortes 


\section{Embellecimiento evolutivo usando el algoritmo de dibujo estético de grafos dirigido por fuerzas}

Esta investigación describe el desarrollo de un algoritmo de embellecimiento facial, que fusiona un algoritmo de trazado estético de grafos con un algoritmo evolutivo. En particular, el operador de mutación del algoritmo evolutivo es implementado a partir de trazo estético dirigido por fuerzas. Con la unión de ambos algoritmos se busca generar un rostro más bello que el rostro de entrada.

Este trabajo toma la idea de enfocar utilizada en [5], esto significa que sólo una característica facial es tomada para realizar el proceso de embellecimiento y el método se concentra únicamente sobre esta. El sistema recibe de entrada una imagen frontal del rostro, junto con sus respectivos puntos de referencia. Los puntos de referencia de la característica seleccionada son llamados puntos seleccionados, el resto son puntos fijos. La Fig. 4(a) muestra el caso de seleccionar la boca como característica a embellecer y en la Fig. 4(b) han sido seleccionadas las cejas para su embellecimiento.

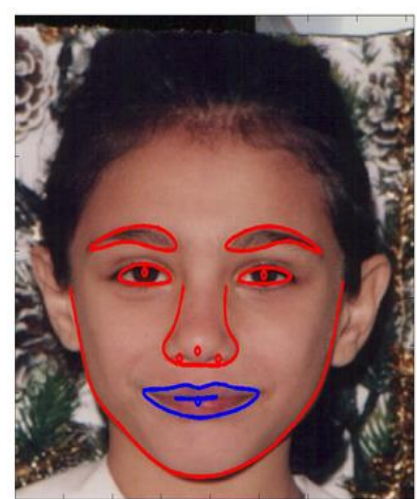

(a)

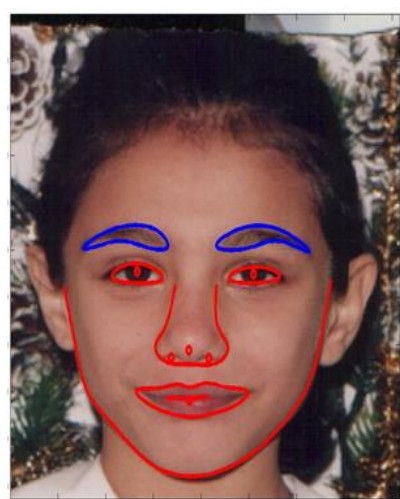

(b)

Fig. 4. Selección de la Característica a Embellecer: a) la boca y b) las cejas

El proceso de embellecimiento se muestra en la Fig. 5. Dada una foto frontal como entrada el usuario selecciona la característica facial a embellecer. A partir de los puntos de referencia, se extrae un vector de distancias, que corresponden a la máscara del rostro de entrada. Esta mascara pasa al Algoritmo Genético, el cual proporciona como resultado un vector de distancias modificado que posee una calificación de belleza más alta que el vector original.

La transformación warping es utilizada para modificar la imagen de entrada, este proceso mapea el conjunto de puntos de referencia $\{p i\}$ de la imagen origen en el correspondiente conjunto de puntos de referencia $\{q i\}$ de destino.

\subsection{Funcionamiento del algoritmo evolutivo}

Un individuo I ésta compuesto por la máscara: Puntos de Referencia y Vector de Distancias. El funcionamiento principal (o ciclo evolutivo) del Algoritmo Evolutivo se realiza de la siguiente forma: 
1. Generar una población inicial de 21 individuos formada por el individuo original $I$ y 20 versiones mutadas generadas utilizando el algoritmo de dibujo estético de grafos dirigido por fuerzas.

2. Evaluar a la población actual.

3. El mejor individuo (es aquel que tiene la mejor calificación proporcionada por la función de evaluación) es seleccionado para ser el primer individuo de la nueva población.

4. Seleccionar 10 padres de la población actual por certamen.

5. Cruzar a los padres seleccionados, los descendientes se incorporan a la nueva población.

6. Utilizar al mejor individuo de la población para generar 10 descendientes mutados haciendo uso del algoritmo de dibujo estético de grafos dirigido por fuerzas. Estos complementan los 21 individuos de la nueva población.

7. Reemplazar la población actual por la nueva población.

8. Volvemos al paso hasta completar el número de generaciones.

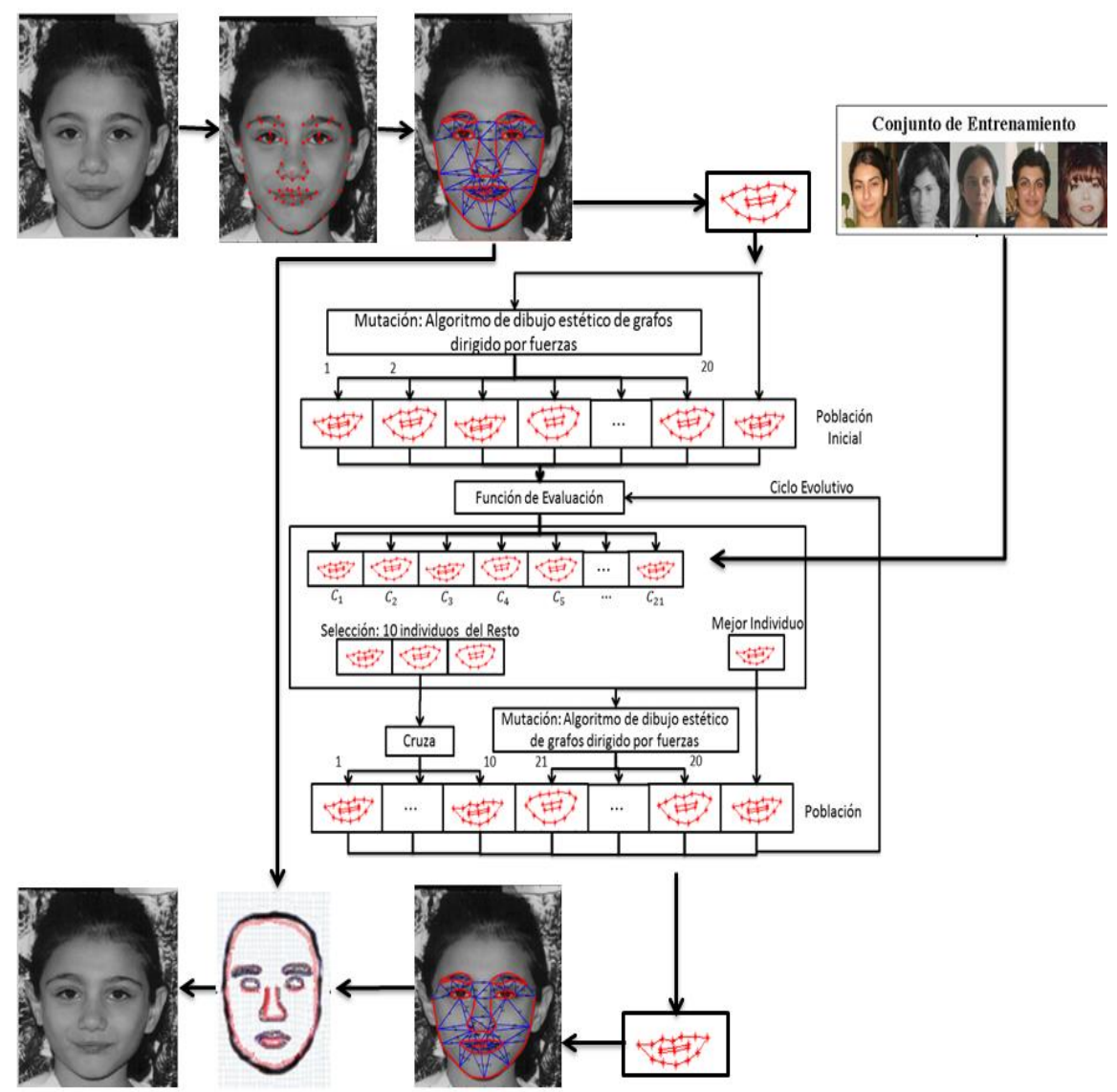

Fig. 5. Procedimiento de Embellecimiento la BOCA como característica a embellecer 


\subsection{Función de aptitud}

La función de evaluación utilizada para evaluar la belleza en esta investigación hace uso de eigenespacios (usados para calificar la belleza en [4], usados en otros trabajos $[5,6,7,8,9])$ los cuales tomando como ensamble vectores de puntos antropométricos de rostros bellos. El Algoritmo Evolutivo genera un vector vecino $v$ ' con una calificación de belleza más alta $\left(v^{\prime}\right)>(v)$, donde $v$ es el vector original.

\subsection{Selección}

La selección de la población para cruce se realiza por certamen: 20 individuos participan en competencias partwise, es decir para cualquier I1 e I2 (elegidos aleatoriamente) que aún no han participado en una competencia, si (I1) > (I2) es seleccionado el Individuo $I$, en caso contrario es seleccionado el Individuo $I 2$.

\subsection{Cruce y mutación}

Los individuos seleccionados (o padres) son cruzados utilizando el método cruce en un punto. Después a partir del mejor individuo mediante mutación se generan 10 individuos utilizando el algoritmo de dibujo estético de grafos dirigido por fuerzas, en este algoritmo es muy importante la determinación de cuatro constantes. $c_{1}, c_{2}, c_{3}$ y $c_{4}$, para poder generar individuos válidos.

\section{Pruebas y resultados}

Los parámetros $c_{1}, c_{2}, c_{3}$ y $c_{4}$ utilizados en el algoritmo de dibujo estético de grafos dirigido por fuerzas son ajustados experimentalmente de acuerdo a sus efectos sobre la visualización de los grafos que forman cada característica del rostro. Por lo anterior se realizaron tres experimentos considerando los parámetros $c_{1}$ y $c_{4}$, que nos permitió ajustar el intervalo de valores que pueden tomar estos.

Los experimentos realizados muestran que los ajustes a los parámetros permiten la generación de grafos validos los cuales se visualizaran de una forma mucho más clara. También en los experimentos identificamos que solo es necesario realizar el ajuste en los parámetros $c_{1}$ y $c_{4}$, esto se atribuye a que el parámetro $c_{1}$ es usado en el cálculo de las fuerzas atractivas (entre el conjunto de nodos que tiene aristas) y el parámetro $c_{4}$ determina la tasa de cambio repulsivo o atractivo. Dichos experimentos se definen a continuación:

Experimento 1: La Fig. 6 muestra seis resultados, tres variando el parámetro $c_{1}$ : (a) $c_{1}=1$, (b) $c_{1}=0.5$, (c) $c_{1}=0.125$, dos variando el parámetro $c_{4}$ : incisos (d) $c_{4}=0.0125$ y (e) $c_{4}=0.0625$. Por último un experimento variando los parámetros $c_{1}$ y $c_{4}$ : es la combinación de variar ambas parámetros (f) $c_{1}=0.125$ y $c_{4}=0.0125$.

En este experimento podemos observar que conforme la iteración incrementa el grafo se deforma en este caso para nuestro problema. Es decir si dejamos constante este parámetro al final se producirían grafos no útiles para nuestro problema. 
Experimento 2: En un experimento 2 se variaron los parámetros $c_{1}$ y $c_{4}$ aleatoriamente, la Fig. 7 muestra algunos resultados variando aleatoriamente los parámetros utilizando el mismo valor del parámetro en cada una de las aristas que conforman el grado de la característica facial.

Este experimento muestra que podemos obtener variabilidad muy rápido debido a la aleatoriedad, pero aun así se puede observar que el grafo solo crece.

Experimento 3: Por último se realizó un tercer experimento en el cual se variaron los parámetros $c_{1}$ y $c_{4}$ aleatoriamente en cada una de las aristas que conforman la el grafo de la característica facial. La Fig. 8 muestra algunos resultados.

Podemos observar en los resultados que la aleatoriedad en cada arista nos permite tener grafos con diferentes formas más variables.

(a)

(b)

(c)

(d)

(e)

(f)

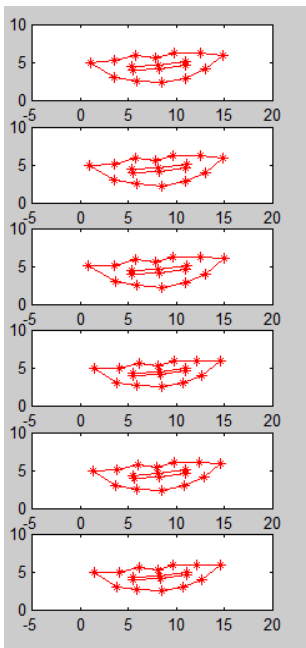

Primer Iteración

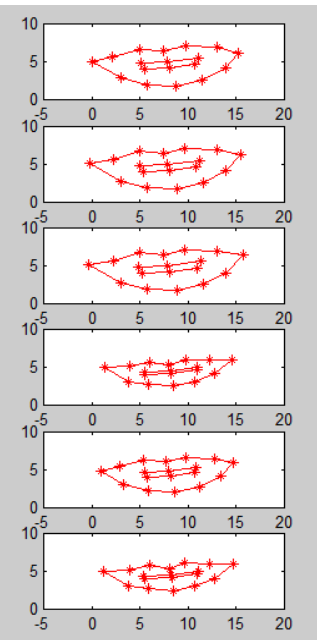

Segunda Iteración

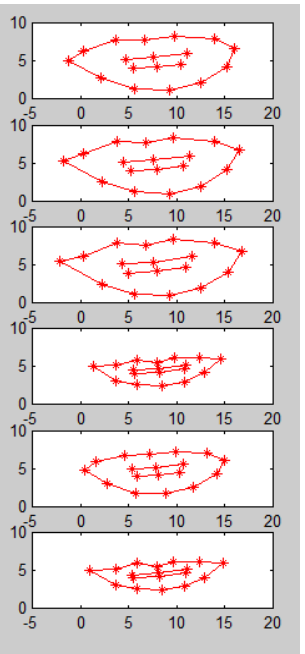

Tercer Iteración

Fig. 6. Experimento 1: Las bocas generadas variando los parámetros $c_{1} y c_{4}$. (a) $c_{1}=1$, (b) $c_{1}=0.5$, (c) $c_{1}=0.125$, (d) $c_{4}=0.0125$, (e) $c_{4}=0.0625$ y (f) $c_{1}=0.125$ y $c_{4}=0.0125$

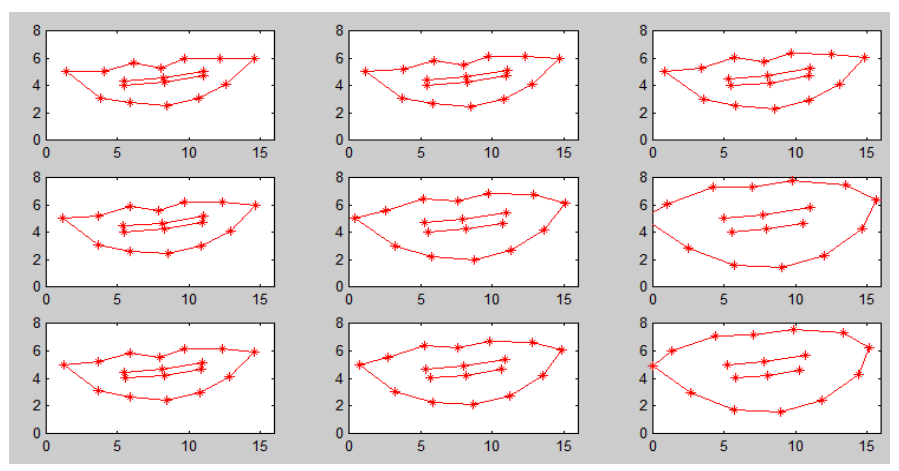

Fig. 7. Experimento 2: Bocas generadas variando los parámetros $c_{1}$ y $c_{4}$ aleatoriamente, pero utilizando estos mismos parámetros en cada una de las aristas que conforman la boca 
Basados en los experimentos y resultados se determinaron los rangos de valores que pueden tomar los parámetros: $c_{1} \in[0.0625,2]$ y $c_{4} \in[0.00625$ y 0.1$]$.

Algunos resultados de embellecimiento evolutivo para diferentes características faciales, utilizando el algoritmo de dibujo estético de grafos dirigido por fuerzas como operador de mutación se observan en la Fig. 9, 10, 11, 12, 13,14.

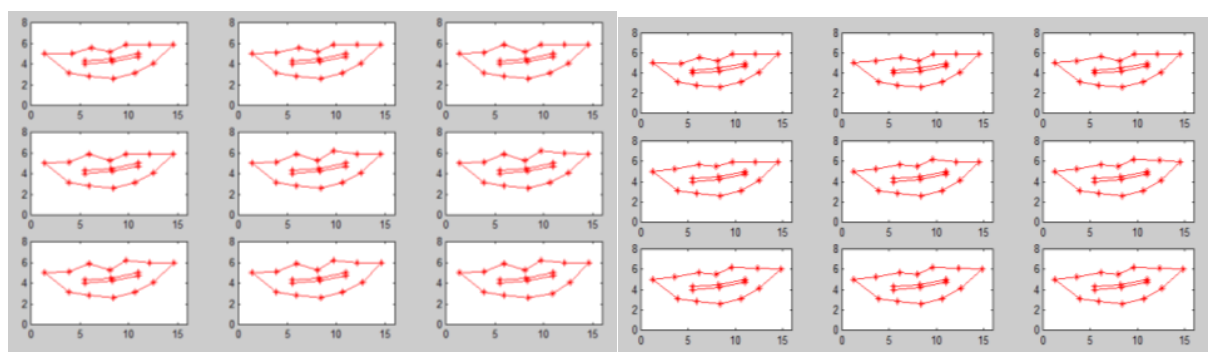

Fig. 8. Bocas generadas variando los parámetros $c_{1}$ y $c_{4}$ aleatoriamente, utilizando diferentes valores del parámetro en cada una de las aristas que conforman la boca
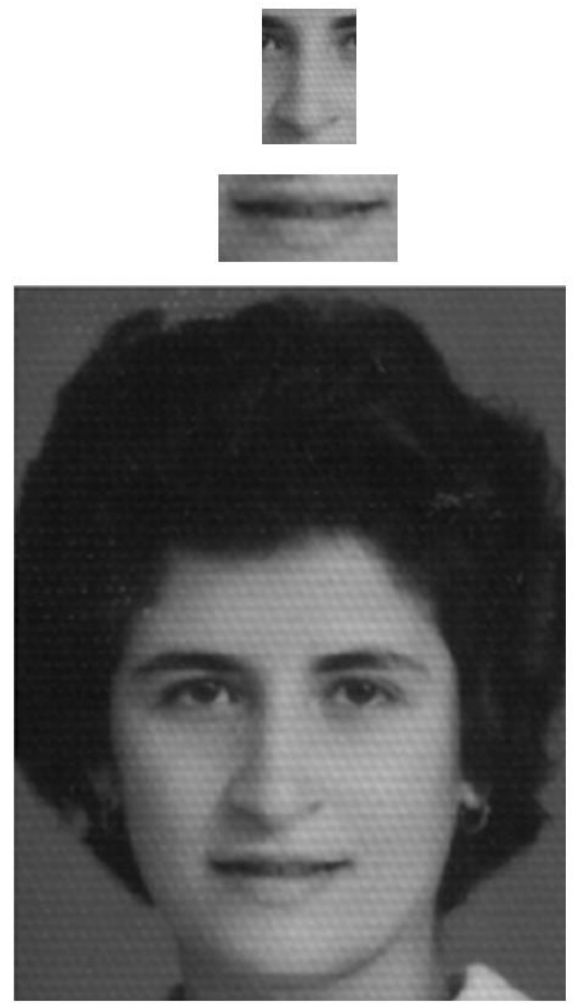
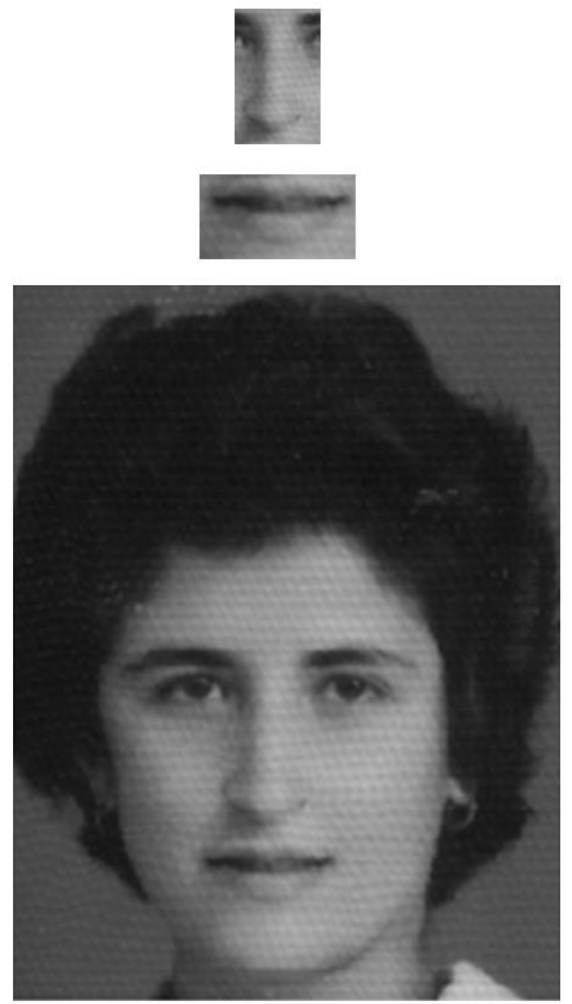

Fig. 9. Resultados de embellecimiento. A empezado a evolucionar las características faciales: "BOCA, CEJAS, NARIZ Y LIMITES DEL ROSTRO". Número de Generaciones 100. Izquierda: rostro original y Derecha: rostro embellecido 
Ricardo Solano Monje, Nayeli Joaquinita Meléndez Acosta, Homero Vladimir Ríos Figueroa
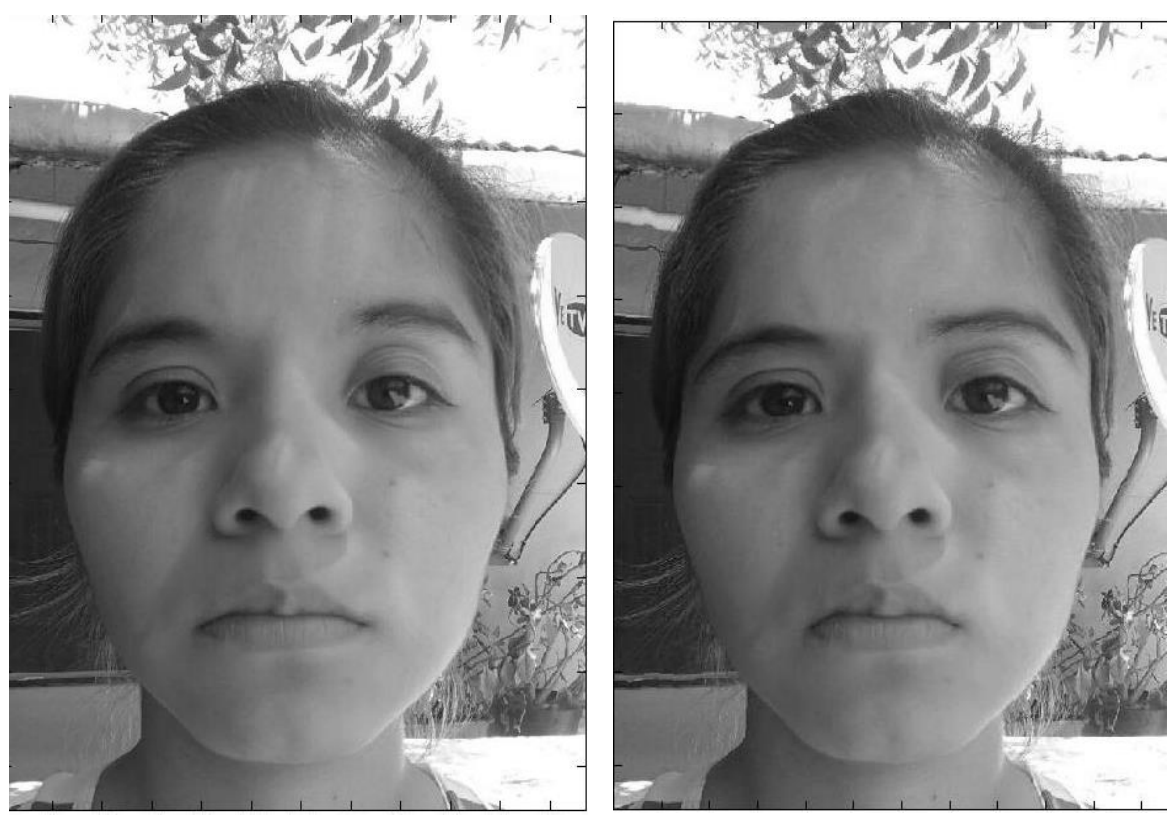

Fig. 10. Resultados de embellecimiento. A empezado a evolucionar las características faciales: "CEJAS, BOCA y OJOS". Número de Generaciones 50. Izquierda: rostro original y Derecha: rostro embellecido
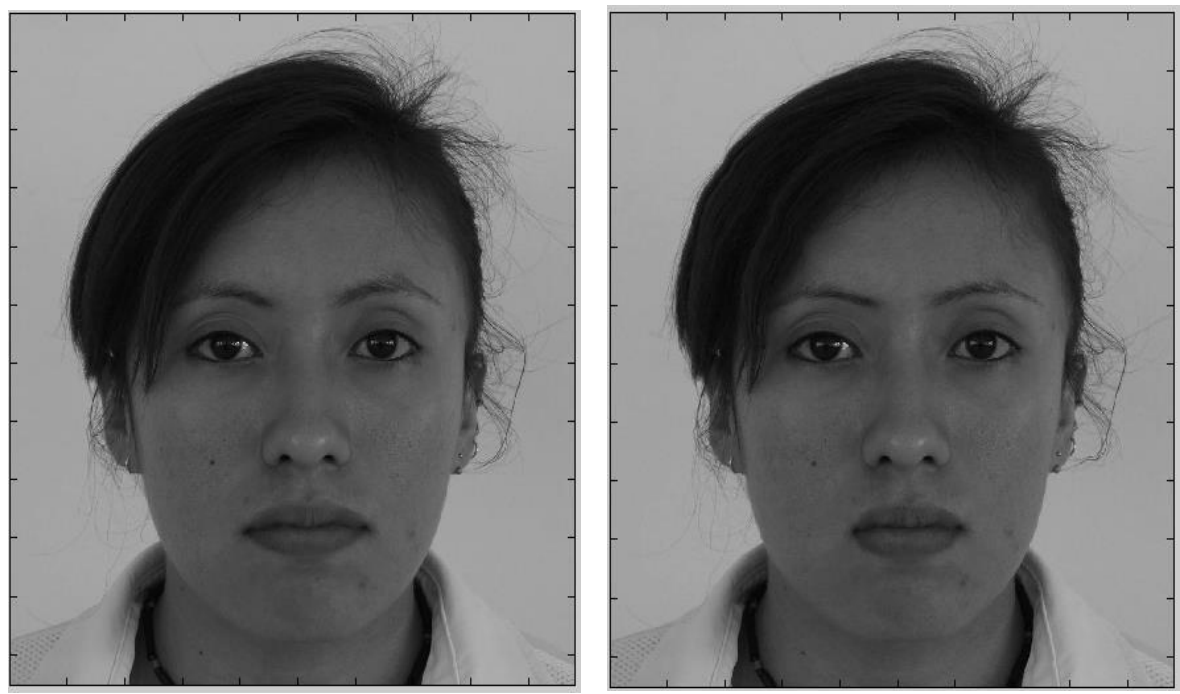

Fig. 11. Resultados de embellecimiento. A empezado a evolucionar las características faciales: “CEJAS, BOCA y LIMITES DEL ROSTRO”. Número de Generaciones 50. Izquierda: rostro original y Derecha: rostro embellecido 

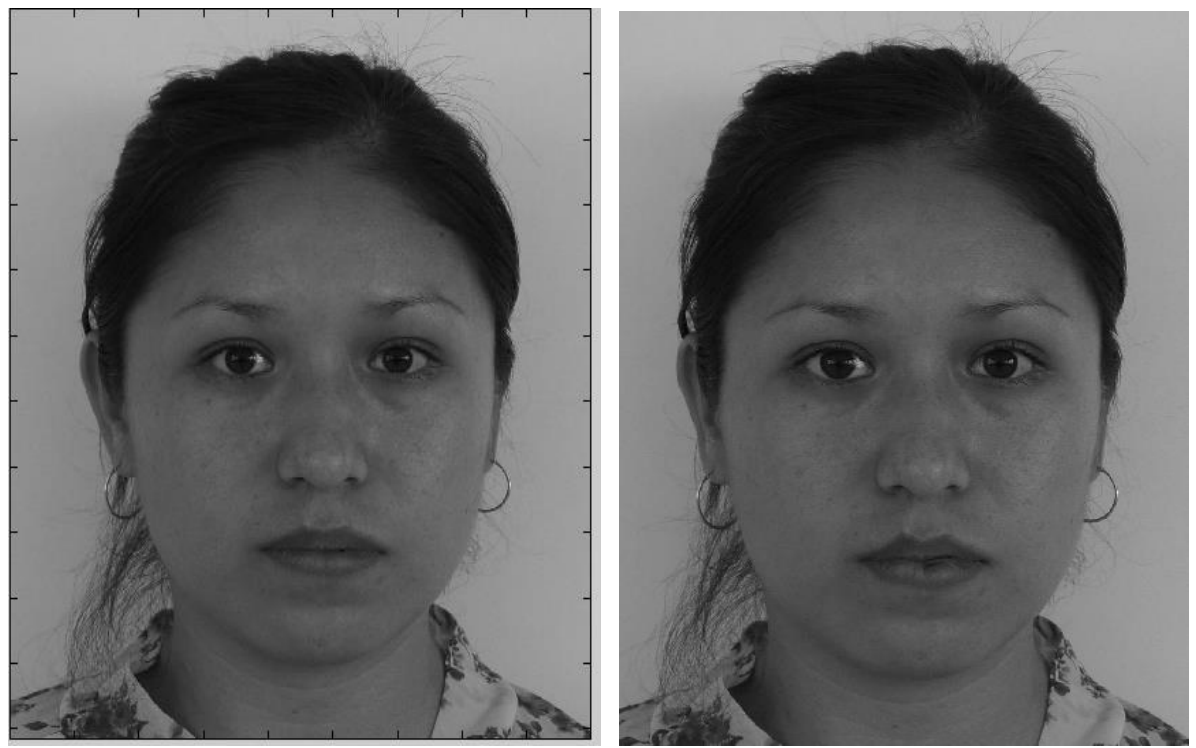

Fig. 12. Resultados de embellecimiento. A empezado a evolucionar las características faciales: "BOCA, CEJAS, BOCA y LIMITES DEL ROSTRO". Número de Generaciones 50. Izquierda: rostro original y Derecha: rostro embellecido

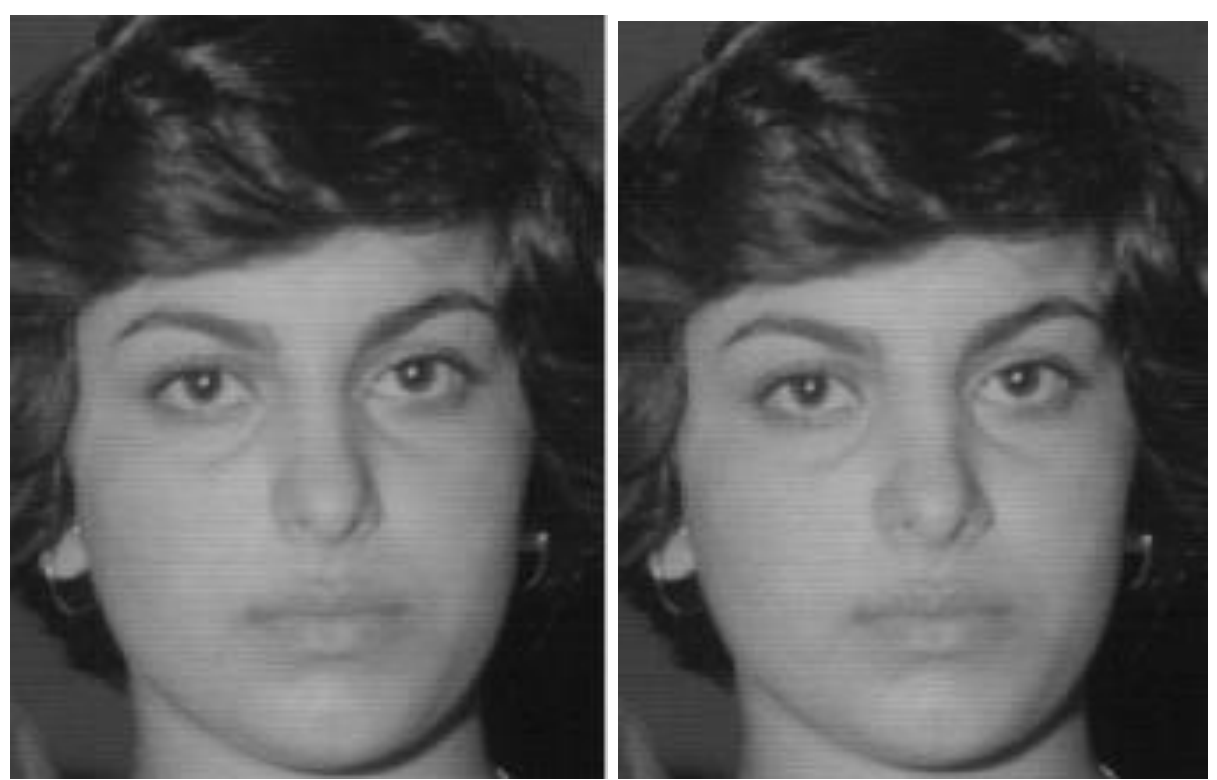

Fig. 13. Resultados de embellecimiento A empezado a evolucionar la característica facial: "NARIZ". Número de Generaciones 50. Izquierda: rostro original y Derecha: rostro embellecido

Para probar el funcionamiento de nuestro sistema se realizó un encuesta a estudiantes de licenciatura a los cuales se les mostro el rostro original junto con el mejorado y ellos indicaron cual es el rostro más bello. Se han considerado 40 
estudiantes para la encuesta, en promedio el $82 \%$ de los estudiantes acertaron cual es la imagen embellecida.
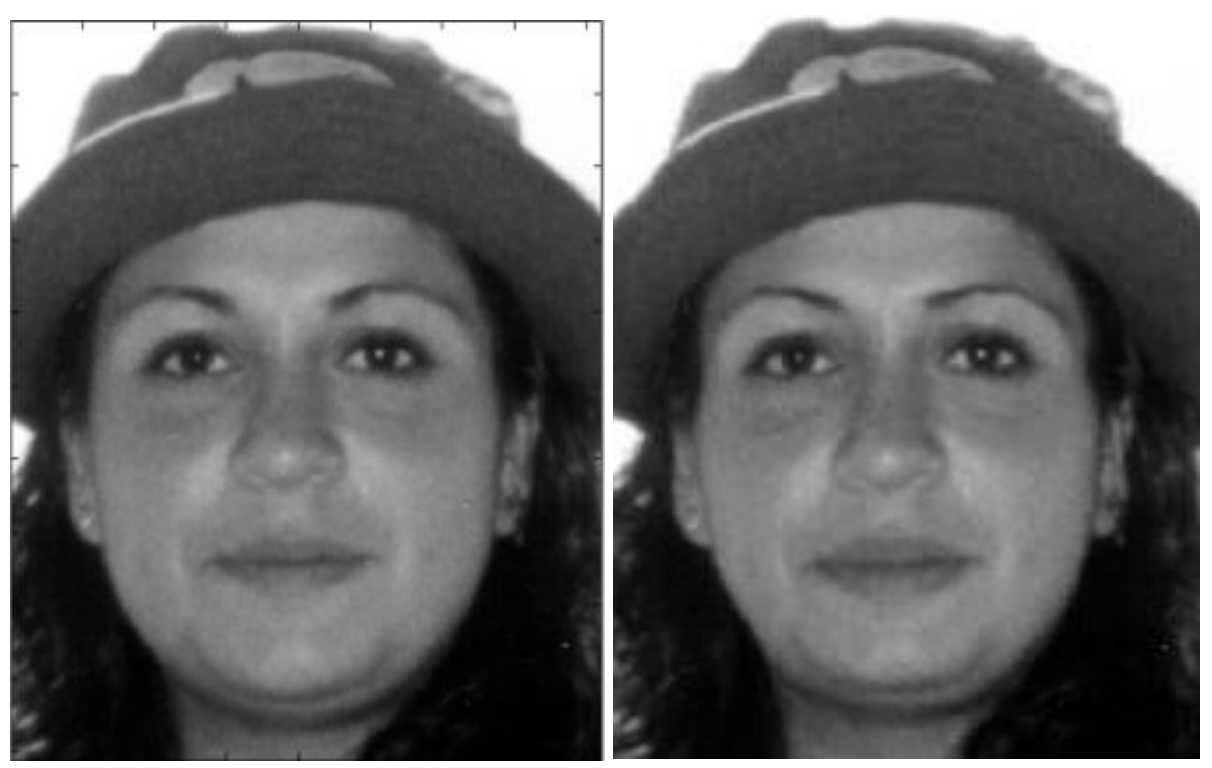

Fig. 14. Resultados de embellecimiento A empezado a evolucionar las características faciales: “BOCA, CEJA y LIMITES DEL ROSTRO”. Número de Generaciones 50. Izquierda: rostro original y Derecha: rostro embellecido

\section{Conclusiones}

Esta investigación describe el desarrollo de un algoritmo de embellecimiento facial, que fusiona un algoritmo de trazado estético de grafos con un algoritmo evolutivo. En particular, el operador de mutación del algoritmo evolutivo es implementado a partir de trazo estético dirigido por fuerzas. Con la unión de ambos algoritmos se busca generar un rostro más bello que el rostro de entrada.

El sistema está limitado a recibir una imagen frontal del rosto junto con sus respectivos puntos de referencia.

Los experimentos realizados permiten terminan los intervalos de valores para los parámetros: $c_{1} \in[0.0625,2]$ y $c_{4} \in[0.00625$ y 0.1$]$.

\section{Referencias}

1. Leyvand, T., Cohen, Or D., Dror G., Lischinski D.: Data Driven Enhancement of Facial Attractiveness. ACM Trans. Graph, Vol. 27, No. 3, p. 9 (2008)

2. Eisenthal, Y., Dror, G., Ruppin, E.: Facial attractiveness: Beauty and the machine. Neural Computation, Vol. 18, No. 1, pp. 119-142 (2006) 
3. Solano Monje, R., Meléndez Acosta, N.J., Juárez Vázquez, S., Rios Figueroa, H.V.: Belleza Artificial: Evolucionando partes del Rostro. Research in Computing Science, Vol. 93, pp. 111-120 (2015)

4. Solano Monje, R., Meléndez Acosta, N.J., Juárez Vázquez, S., Rios Figueroa, H.V.: Eigenespacios de Belleza Paramétricos como Máquina Calificadora. Research in Computing Science, Vol. 93, pp. 133-140 (2015)

5. Murase, H., Nayar, S.K.: Parametric Eigenspace Representation for Visual Learning and Recognition. In: Proceedings of The International Society for Optical Engineering (SPIE), Vol. 2031, pp. 378-391 (1993)

6. Nayar, S.K., Murase, H., Nene, S.A.: Parametric Appearance Representation. In: Early Visual Learning, pp. 131-160 (1996)

7. Nayar, S.K., Baker, S., Murase, H.: Parametric Feature Detection. In: DARPA Image Understanding Workshop (IUW), pp. 1425-1430 (1997)

8. Pentland, A., Moghaddam, B., Starner, T.: View-Based and Modular Eigenspaces for Face Recognition. In: IEEE Conference on Computer Vision \& Pattern Recognition (1994)

9. Solano Monje, R.: Modelado y reconocimiento de objetos usando apariencia y multieigenespacios particionados. Instituto Nacional de Astrofísica, Óptica y Electrónica, Septiembre (2002)

10. Kobourov, S.G.: Unit 12: Force-Directed Drawing Algorithms. University of Arizona. (2004)

11. Utech, J., Branke, J., Schmeck, H., Eades, P.: An Evolutionary Algorithm for Drawing Directed Graphs. In: Internacional Conference on Imaging Science, Systems, \& Technology (CISST'98), pp. 154-160 (1998)

12. Cootes, T.: The Fg-Net Aging Database, http://sting.cycollege.ac.cy/ alanitis/ fgnetaging/index.htm 